\title{
Influence of angled dispersion gas on coaxial atomization, spray and flame formation in the context of spray-flame synthesis of nanoparticles
}

\author{
M. Bieber ${ }^{1} \cdot$ M. Al-Khatib ${ }^{1} \cdot$ F. Fröde ${ }^{2} \cdot$ H. Pitsch ${ }^{2} \cdot$ M. A. Reddemann ${ }^{1} \cdot$ H-J. Schmid ${ }^{3} \cdot$ R. Tischendorf $^{3} \cdot$ R. Kneer $^{1}$
}

Received: 26 November 2020 / Revised: 24 March 2021 / Accepted: 26 March 2021 / Published online: 17 April 2021

(C) The Author(s) 2021

\begin{abstract}
Liquid atomization determines the initial conditions for flame formation and particle synthesis. Without a stable flame, high droplet velocities and thus short droplet residence time in the flame may lead to droplets being injected into an extinguished flame, which influences synthesis and final particle output. An experimental investigation of spray formation and flame stability is performed through high-speed visualization. Targeted variation of nozzle geometry is applied to improve sprayflame interaction and compared to a standardized burner. Timescales of spray density and flame fluctuations are quantified and compared, where the latter were significantly larger and hence not correlated. Instead, dispersion gas forms a barrier between spray phase and pilot flame; hence, ignition depends on large liquid lumps with high radial momentum to break through the dispersion gas for spray ignition. Angling of dispersion gas flow increases radial shear and turbulence and leads to refined atomization and improved flame stability. To investigate the nozzle influence on particle formation, particle characteristics are examined by online and offline analytics with focus on particle structures and product purity. The modified nozzle produced smaller primary particle sizes, thus indicating a sensitivity of sintering dominance on the nozzle geometry. Impurities impact the examination of particle structures and general particle functionality. Carbon contamination was apparent in synthesized particles and also indicated sensitivity to nozzle geometry. Discrepancies to literature data are discussed regarding differences in flame activity and droplet characteristics. The report highlights, how product characteristics can differ crucially due to changes in nozzle geometry despite comparable operating conditions.
\end{abstract}

M. Bieber

bieber@wsa.rwth-aachen.de

1 Institute of Heat and Mass Transfer, RWTH Aachen University, Augustinerbach 6, 52062 Aachen, Germany

2 Institute for Combustion Technology, RWTH Aachen University, Templergraben 64, 52062 Aachen, Germany

3 Particle Technology Group, Paderborn University, Warburger Straße 100, 33098 Paderborn, Germany 


\section{Graphic abstract}

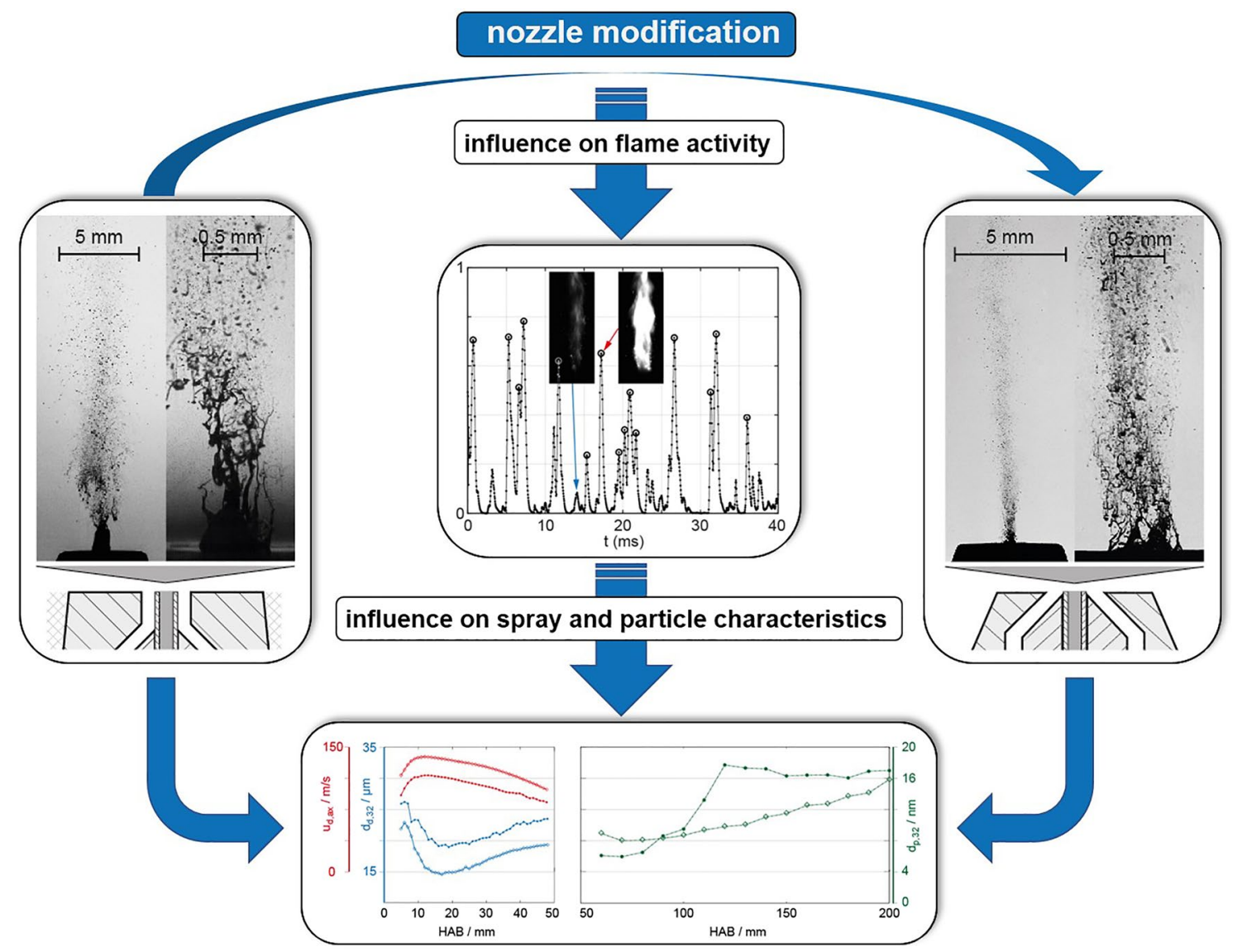

\section{Introduction}

Spray-flame synthesis of nanoparticles is known for its flexibility in producing a wide range of nanoparticle powders with high purity (Strobel and Pratsinis 2007). Produced nanomaterials can consist of metals as well as single metal oxides and more complex mixed oxides Thiebaut (2011) that are utilized in, e.g., catalysts, electroceramics (fuel cells, batteries), medicine (bio materials, diagnostics, drug delivery) or sensors (Andronescu 2017) with superior functionality over conventional products (Strobel and Pratsinis 2007). To exploit the full potential of spray-flame synthesis towards sophisticated product output with unique functionalities, a fundamental understanding of the underlying process chain is required (Stark and Pratsinis 2002). However, the overall process complexity in the flame is very high: particle characteristics are determined in the flame within a few milliseconds residence time and influenced by various sub-processes including atomization and spray formation, droplet evaporation, flame chemistry and the particle formation (Kammler et al. 2001). Although flame spray pyrolysis (FSP) has been already widely focused in research in the past, the links between all subprocesses are not yet fully understood. A commonly observed property of spray flames is flame instabilities which are caused by acoustics, burner geometry and the combustion process (Harvazinski et al. 2013). With the focus on combustion, flame fluctuations may originate in vapor-to-liquid fuel-ratio fluctuations (Greenberg and Cohen 1997 ) as well as initial fuel-rich or fuel-lean gas-phase conditions that influence the resulting flame front propagation (Greenberg et al. 2001). In addition, if the rate of fuel mass consumption exceeds fuel evaporation rate, the flame is prone to fuel starvation which can ultimately lead to flame extinction.

Various viable burner types for particle synthesis can be found in the literature, including diffusion flame, McKenna flat flame, Hencken flat flame or twin fluid atomizer approaches ( $\mathrm{Li}$ et al. 2016). The focus of this work is the synthesis of nanoparticles in spray flame based burner types, i.e., coaxial atomization of liquid precurser and injection 
in a premixed pilot flame, also known as (i) flame spray pyrolysis (FSP) (Sokolowski et al. 1977; Teoh et al. 2010) or (ii) spray-flame synthesis (Stark and Pratsinis 2002; Menser et al. 2015). While the coaxial alignment of dispersion gas flow is characteristic for this type of synthesis, multiple nozzle configuration approaches have been introduced in the past. These range from custom twin fluid atomizers with parallel co-flow of the dispersion gas relative to the liquid flow (Menser et al. 2015), angling of the dispersion gas flow towards the center of the liquid jet (Mädler et al. 2002), or utilization of commercially available atomizers with inclined annular dispersion gas flow and internally imposed swirl to the gas flow (Gröhn et al. 2014). The influence of dispersion gas angle with varying swirl angles on the spray has been investigated by Meierhofer et al. (2014) for hollow cone water jets. Therein, increased swirl angles lead to larger droplets and reduced axial velocity while the spray is stabilized. However, despite being placed in the context of FSP, no results are presented for synthesis conditions and, thus, for the influence on flame behavior and finally particle formation. In general, instabilities and flame fluctuations may lead to significant temporal local change of particle synthesis conditions inside the flame. Therefore, a strong impact of fluctuations on the final nanoparticle properties is expected, as, e.g., a broadening of the particle size distribution. The extent and characteristics of flame fluctuations significantly depend on the local conditions (composition, velocity, temperature) inside the reaction zone.

In this context, the atomization process at the nozzle outlet can be assumed to have a major influence on the formation of local flame conditions. For this reason, this work is dedicated to the investigation of the influence of atomization and nozzle design on flame formation and stability. Atomization of a liquid jet by an annular gas stream generally occurs via two pathways: The primary breakup of ligaments at the circumference of the jet or the secondary breakup of liquid lumps at the end of the jet (Lasheras et al. 1998). Especially at moderate operation conditions, the primary breakup mechanism is predominant, which is characterized by growth of primary (Kelvin-Helmholtz) and secondary (Rayleigh-Taylor) instabilities and consequently formation and elongation of liquid ligaments that ultimately disintegrate into droplets (Marmottant and Villermaux 2004; Bieber et al. 2017). The near-field development of liquid jets is characterized by the jet Reynolds number $\operatorname{Re}_{1}=u_{1} \cdot D_{1} / v_{1}$ with liquid jet exit velocity $u_{1}$, outer capillary diameter $D_{1}$ and kinematic viscosity $v_{1}$. Another highly relevant nondimensional number for the jet breakup is the initial aerodynamic Weber number $\mathrm{We}_{0, \mathrm{~g}}=\rho_{\mathrm{g}} \cdot u_{\mathrm{rel}}^{2} \cdot D_{\mathrm{l}} / \sigma$ as well as the gas-to-liquid momentum flux ratio $\mathrm{M}=\rho_{\mathrm{g}} \cdot u_{\mathrm{g}}^{2} / \rho_{1} \cdot u_{1}^{2}$ (Varga et al. 2003), with liquid/gas density $\rho$, relative velocity between liquid and gas-phase $u_{\text {rel }}^{2}$ and surface tension $\sigma$.
With increasing dispersion gas flow, a major part of droplets are formed by shedding of liquid lumps directly from the initial core jet that further disintegrate into droplets due to secondary atomization (Lasheras et al. 1998). This shedding occurs at a characteristic frequency which depends in particular on the axial gas velocity and resulting momentum flux ratio. In this context, secondary breakup downstream of the liquid core may account for a considerable fraction of the liquid atomization and thus determines the final droplet size distribution of the spray (Lasheras and Hopfinger 2000).

\section{Materials and method}

For the spray flame, a standardized burner is used (Menser et al. 2015; Schneider et al. 2019) (so-called SpraySyn burner $^{1}$ ) is utilized. As illustrated in Fig. 1 right, the liquid jet is atomized by a coaxial dispersion gas stream. Ignition of the resulting flammable mixture is conducted by a co-axially arranged pilot flame.

To ensure a constant liquid feed, a dual rate syringe pump (KD Scientific, type: Gemini 88) is utilized. The pilot flame is generated by directing premixed $\mathrm{CH}_{4}$ and $\mathrm{O}_{2}$ at a oxidator-fuel ratio $\lambda=4\left(V_{\mathrm{CH} 4}=2 \mathrm{slm}, V_{\mathrm{O} 2}=16 \mathrm{slm}\right)$ through a porous sintered brass matrix, paired with a pressured sheath air co-flow of $V_{\text {air }}=120 \mathrm{slm}$ for matrix cooling and spray flame confinement.

All measurements in this work are performed with a precursor solution of dissolved iron(III)nitrate nonahydrate ( $\geq 99 \%, 0.1 \mathrm{~mol} / \mathrm{l}$ ) in a solvent mixture of $35 \%$ vol ethanol and $65 \%$ vol 2-ethylhexanoic acid (EHA, $\geq 99 \%$ ). Liquid material properties have been determined by Bieber et al. (2019) to: density $\rho=867.83 \mathrm{~kg} / \mathrm{m}^{2}$, kinematic viscosity $v=4.44 \cdot 10^{-6} \mathrm{~m}^{2} / \mathrm{s}$, surface tension $\sigma=2.57 \cdot 10^{-6} \mathrm{~N} / \mathrm{m}$. Pure oxygen is used as dispersion gas. All gas feeds are provided by Bronkhorst EL-FLOW SELECT F-201AV mass flow controllers. The burner is operated with corresponding standard operation conditions: $V_{1}=2 \mathrm{ml} / \mathrm{s}$ liquid feed rate and $V_{\mathrm{g}}=10 \mathrm{slm}$ dispersion gas (Schneider et al. 2019). Resulting fluid velocities and relevant dimensionless quantities are presented in Table 1.

The burner is embedded into a semi-confined hexagonal combustion chamber with full optical access (Bieber et al. 2019). For analysis of spray and flame, high-speed shadowgraphy measurements of the flame and droplet phase are conducted with a combination of varying illumination

\footnotetext{
1 The SpraySyn burner has been developed for the German Research Foundation priority program SPP1980 for synthesis of nanoparticles (Menser et al. 2015; Schneider et al. 2019). Standardized operation parameters are utilized in this work for cross comparison of experimental and numerical results of other SPP1980 participants. All measurement data are provided to on an open source project database.
} 


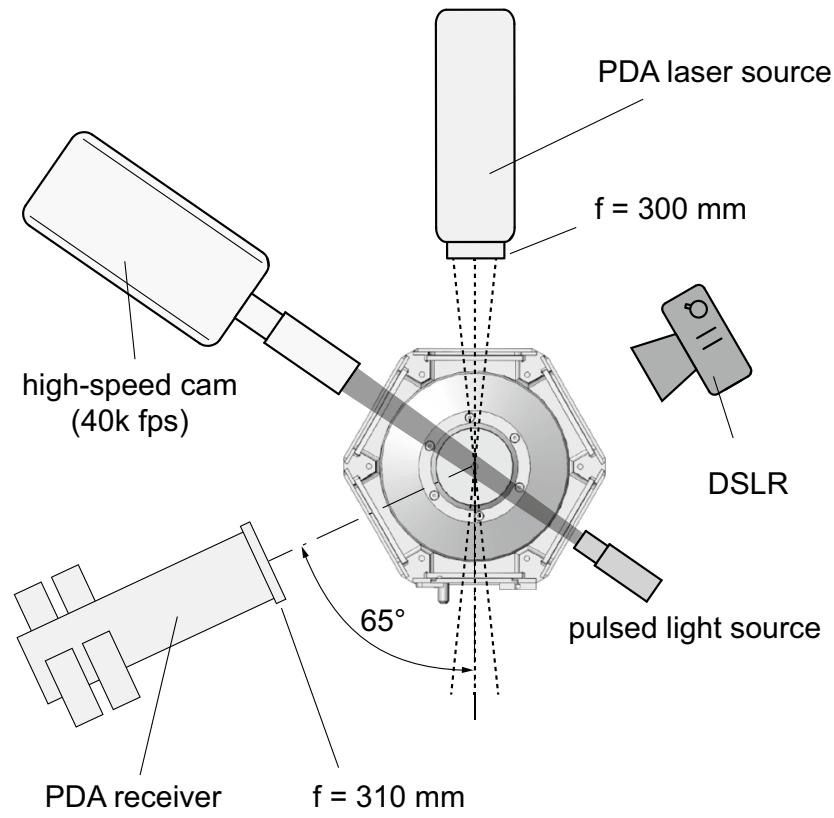

Fig. 1 Left: Schematic of the general test setup. Right: Geometry (top) and schematic illustration of the SpraySyn burner (bottom) (Menser et al. 2015). Liquid feed is injected through a centered cannula and atomized with a coaxial $\mathrm{O}_{2}$ dispersion gas flow. The pilot

Table 1 Standard operating conditions of the SpraySyn burner $\left(V_{\mathrm{g}}\right.$ $=10 \mathrm{slm}, V_{\mathrm{l}}=2 \mathrm{ml} / \mathrm{min}$ ) (Schneider et al. 2019)) and corresponding dimensionless quantities at nozzle exit for coaxial atomization

\begin{tabular}{lllll}
\hline $\begin{array}{l}\mathrm{U}_{\mathrm{g}} \\
(\mathrm{m} / \mathrm{s})\end{array}$ & $\begin{array}{l}\mathrm{U}_{\mathrm{l}} \\
(\mathrm{m} / \mathrm{s})\end{array}$ & $\begin{array}{l}\mathrm{Re}_{1} \\
(-)\end{array}$ & $\begin{array}{l}\mathrm{We}_{\mathrm{g}} \\
(-)\end{array}$ & $\begin{array}{l}\mathrm{M} \\
(-)\end{array}$ \\
\hline 120.6 & 0.27 & 40.1 & 553.6 & 326.7 \\
\hline
\end{tabular}

techniques and magnification (Camera: Photron Fastcam SA-X, 30k fps at $768 \times 376$ pixel resolution, $33 \mu$ s exposure time). Qualitative high-speed images of the flame are obtained by capturing the resulting particle glow in the flame without background illumination with a $100 \mathrm{~mm}$ macro lens (Laowa $100 \mathrm{~mm} \mathrm{f/2.8} \mathrm{2 \times} \mathrm{Ultra} \mathrm{Macro} \mathrm{APO).}$ Simultaneous capturing of flame glow and droplet phase is obtained by using maximum camera exposure time (1/frame rate $=\Delta \mathrm{t}_{\text {exp }}=33 \mu \mathrm{s}$ ) for capturing of flame glow in combination with short pulsed background illumination for droplet shadowgraphy. To minimize motion blur, a monochromatic and incoherent pulsed light source (Cavilux Smart, $640 \mathrm{~nm}$ wavelength, $10 \mathrm{~ns}$ pulse duration) is used for freezing the droplet phase while the long camera exposure time enables capturing visible light emissions from the flame. Hence, the final image contains an instantaneous shadowgraphy footprint of the droplet phase during of the $10 \mathrm{~ns}$ time interval of background illumination as well as an integral image of light emission from the flame during $\Delta \mathrm{t}_{\exp }=33 \mu \mathrm{s}$. In addition,

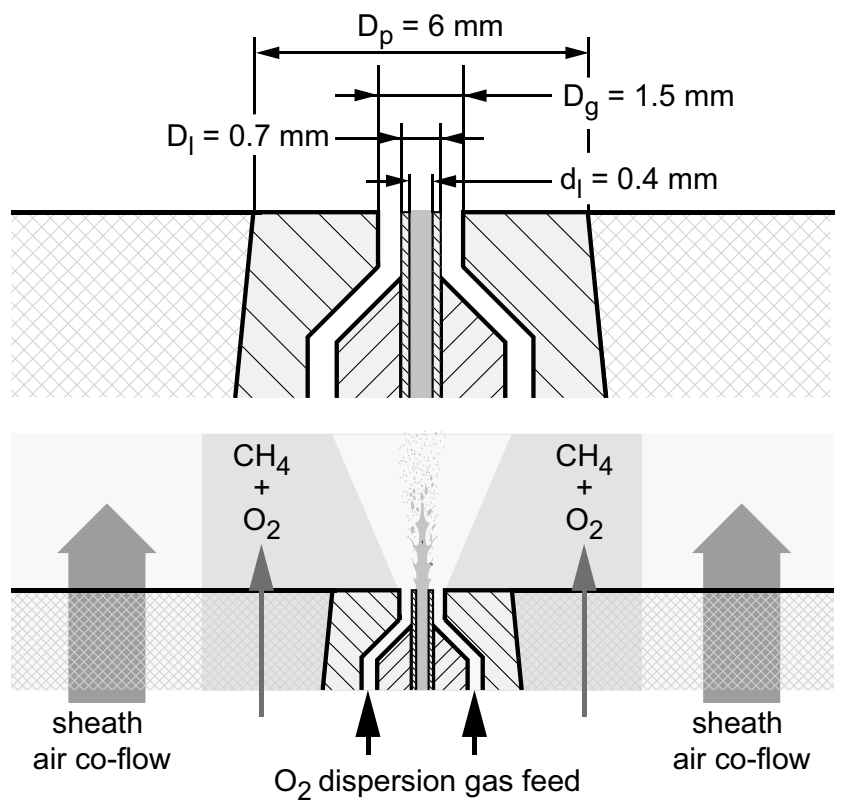

flame is generated by directing premixed $\mathrm{CH}_{4}$ and $\mathrm{O}_{2}$ at $\lambda=4$ through a porous sintered brass matrix. The resulting spray flame is confined by a dual purpose pressured air co-flow that is also directed through the sinter for cooling

microscopic high-speed shadowgraphy is performed to capture primary atomization at nozzle exit $(30 \mathrm{k} \mathrm{fps}$ at $768 \times 376$ pixel resolution, $33 \mu$ s exposure time, Lens 2 : Navitar $8 \times$ Mag Zoom Lens, Cavilux Smart, $640 \mathrm{~nm}$ wavelength, $10 \mathrm{~ns}$ pulse duration background illumination). Note, flame glow can exclusively be captured in macroscopic images. For high magnification, flame light emission is not sufficient for illumination of the camera sensor and background illumination is dominated by the light source.

Droplet sizes are determined with phase-Doppler technique (laser source: ILA 2D fp50 LDV System with $250 \mathrm{~mW}$ at $532 \mathrm{~nm}$ and $561 \mathrm{~nm}$; receiver: DANTEC PDA Classic, BSA P80 processor). The receiver is positioned for observation at scattering angle of $65^{\circ}$ in order to minimize the influence of potential refractive index variation in the flame caused by evaporation of more volatile components in the solvent mixture (Bieber et al. 2019); Pitcher and Wiley (1991). The combustion chamber is mounted on a combination of three linear translation stages that move in $x, y$ and $z$-direction with a minimum increment of $0.1 \mathrm{~mm}$. The spray flame is scanned in axial direction at specific heights above the burner.

To investigate the influence of nozzle variation on particle formation, particle characteristics are examined in two ways. First, the final particle outcome is examined by an offline powder characterization. Second, particles were extracted in situ by means of a hole in a tube (HIAT) probe and their agglomerate/aggregate sizes are subsequently measured by 
online scanning mobility particle sizing (SMPS). The final powder properties are evaluated by mass spectrometry coupled thermogravimetric analysis (TGA-MS) and BrunauerEmmet-Teller (BET) physisorption method (Brunauer et al. 1938). The necessary amount of powder product is derived by surface filtration of the synthesis aerosol. For further details on the particle filtration setup, see Tischendorf et al. (2020). Due to BET measurements, values for the powders Specific Surface Area (SSA) are determined by $\mathrm{N}_{2}$ adsorption. Corresponding measurements are conducted on a Quanta-chrome Autosorb 6 at $-196^{\circ} \mathrm{C}$ after a vacuum sample degassing at $120^{\circ} \mathrm{C}$ over night. Subsequently, the surface equivalent BET primary particle diameter $d_{P, B E T}$ is calculated by a corresponding iron oxide crystallite solid density of $5.24 \mathrm{~g} / \mathrm{cm}^{3}\left(\gamma-\mathrm{Fe}_{2} \mathrm{O}_{3}\right.$, Gestis database, ZVG ref. 1860). TGA-MS measurements are conducted under air, and samples are heated by a temperature ramp of $10 \mathrm{~K} / \mathrm{min}$ until reaching a complete mass steady state (Mettler Toledo SDTA851). During the thermal treatment, evolved gases are detected by the MS at mass to charge $\mathrm{m} / \mathrm{e}$ ratios of 18 and 44 corresponding to the emission of water vapor and $\mathrm{CO}_{2}$. The evolution of agglomerate and aggregate sizes along the center flame axis is examined by the HIAT particle extraction. Subsequently the particles electrical mobility size equivalent diameters are determined using a $2 \mathrm{~nm}$-SMPS setup (TSI Inc., scan range from 1.54 to $48.7 \mathrm{~nm}, 60 \mathrm{~s}$ scan). The probe is adjusted in downstream orientation consisting of a stainless steel tube $\left(d_{\text {tube }}=10 \mathrm{~mm}\right)$ with a corresponding sampling orifice diameter of $d_{\text {orifice }}=1 \mathrm{~mm}$. For more information, see Tischendorf et al. (2020). To ensure a representative extraction, samples are diluted until agglomeration during extraction is negligible (measured mobility distributions remains unchanged despite increasing dilution ratios). This way, the particle number concentrations in the diluted probe correspond to values around $10^{5} \# / \mathrm{mL}$ (measured by means of an additional CPC, model 3775, TSI Inc.). Although sufficient dilution ratios are applied, values for the dilution ratios are not presented as ex situ calibration approaches for this probe are not expected to suit well for the in situ extraction (Tischendorf et al. 2020).

\section{Results}

To analyze the potential influence of fluctuations in the droplet phase on flame activity, high-speed images of the flame and droplet phase have been recorded simultaneously, and subsequently compared as exemplarily shown in Fig. 2. Two contrasting flame states can be identified in a): left, a flame state with strong light emission from particle glow that is considered as an active flame and right, an extinguished flame with too low or without light emission.
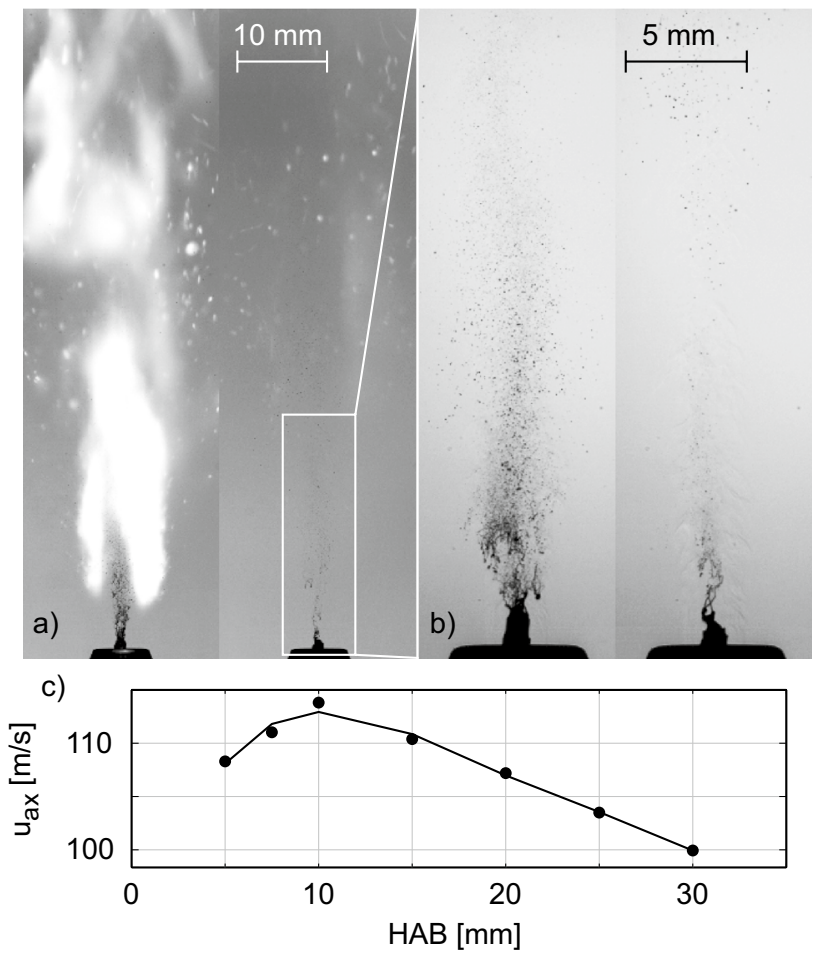

Fig. 2 Instantaneous spray flame images with identical injection parameters and varying image acquisition techniques. a Spray phase shadowgraphy images with flame activity captured at different times with high flame activity (left) and low activity (right). b Spray phase shadowgraphy at different times with high (left) and lower spray density (right). c Mean axial center line droplet velocity in dependency on height above burner (HAB)

Hence, the high-speed videos show a highly in-stationary flame behavior with characteristic repeating sequences of ignition, combustion, extinction and re-ignition. Such flame fluctuations will have a direct influence on the local reaction conditions that the synthesized particles experience on their trajectory through the flame. In this context, the question arises if the reaction conditions already change during the residence time of single particles or if almost constant flame conditions can be assumed for single particles. To answer this question, a rough comparison of the characteristic timescale of flame fluctuations with the residence time of the particles inside the flame shall be conducted. For this purpose it is assumed that the residence time of the droplets in the flame is a characteristic measure for residence time of particles, since the droplets in the relevant region of particle formation are small and follow the gas phase. Laser Doppler velocimetry (LDV) method is used to determine droplet velocities (Bieber et al. 2019). In Fig. 2c, corresponding mean axial droplet velocity in the spray center is presented in dependency on height above burner (HAB). From 5-10 mm, the droplet phase accelerates to a maximum value of $114 \mathrm{~m} / \mathrm{s}$ at $\mathrm{z}=10 \mathrm{~mm}$ before it decelerates again 
Fig. 3 a Approximation of droplet mass fluctuations via normalized mean gray level evaluation $\left(\mathrm{NMG}_{\mathrm{d}}\right)$ of binarized images from a spray section between 5-8 $\mathrm{mm}$ above burner. b Approximation of flame activity via sectional normalized mean gray level evaluation of particle glow $\mathrm{NMG}_{\mathrm{f}}$ between 15-30 mm above burner. c $\mathrm{NMG}_{\mathrm{d}}$ of the selected spray section (cf. a)) over time. $\mathbf{d ~ N M G}_{\mathrm{f}}$ of the selected flame section (cf. b)) over time. $\mathbf{e} \mathrm{NMG}_{\mathrm{f}}$ of the selected flame section over time of configuration $\mathrm{C}$ for qualitative comparison

due to air entrainment and free jet relaxation. However, up to $\mathrm{z}=30 \mathrm{~mm}$, mean droplet velocity remains above $100 \mathrm{~m} / \mathrm{s}$. Under the simplifying assumption of linear acceleration, mean axial droplet phase velocity reaches its maximum after $\Delta \mathrm{t}<0.2 \mathrm{~ms}$ at $\mathrm{z}=10 \mathrm{~mm}$. Assuming a linear trend of deceleration and an average velocity of $100 \mathrm{~m} / \mathrm{s}$, droplets will have passed through the flame after $\Delta \mathrm{t} \approx 0.7 \mathrm{~ms}$ for the given flame height $\mathrm{h}=70 \mathrm{~mm}$, which means that droplet residence time intervals within the flame are potentially smaller than time intervals of flame fluctuations. Consequently, droplets and synthesized particles would be exposed to different but quasi-steady reaction conditions: active flame, extinguished flame or intermediate states. As stated above, this results in inconsistent boundary conditions for evaporation, combustion and ultimately particle synthesis and presumably leads to a broadening in the final size distribution of produced nanoparticles.

High flame activity requires sufficient droplet evaporation and therefore free droplet surface. Complementary videos to Fig. 2a indicate that the droplet density and the integral droplet surface area (represented by the amount and size of droplets propagating in axial direction) also fluctuate over time and that high amounts of droplets initiate flame ignition. Fluctuation of free droplet surface is further emphasized in Fig. 2b, in which two exemplary images are shown that indicate a significant difference in the droplet quantity and hence projected droplet surface area over time.

\subsection{Quantification of spray and flame fluctuations}

To quantify possible fluctuations of the free droplet surface or spray density, a predefined section of the spray between $5 \mathrm{~mm}<\mathrm{HAB}<8 \mathrm{~mm}\left(7<\Delta \mathrm{z} / \mathrm{D}_{1}<11.5\right)$ is analyzed. In this spatial section, primary atomization is completed and only dispersed droplets are visible, as exemplarily shown in Fig. 3a. Data evaluation is conducted based on 5000 consecutive binarized images of a time-series that is captured at $30 \mathrm{k}$ fps. Thereby, the normalized projected cross-sectional area of passing droplets as well as the normalized mean gray level (NMG-normalized with the maximum NMG value) of the entire section has been determined with identical outcome. In the following, the NMG is considered further for data evaluation due to advantages with regard to computational cost.
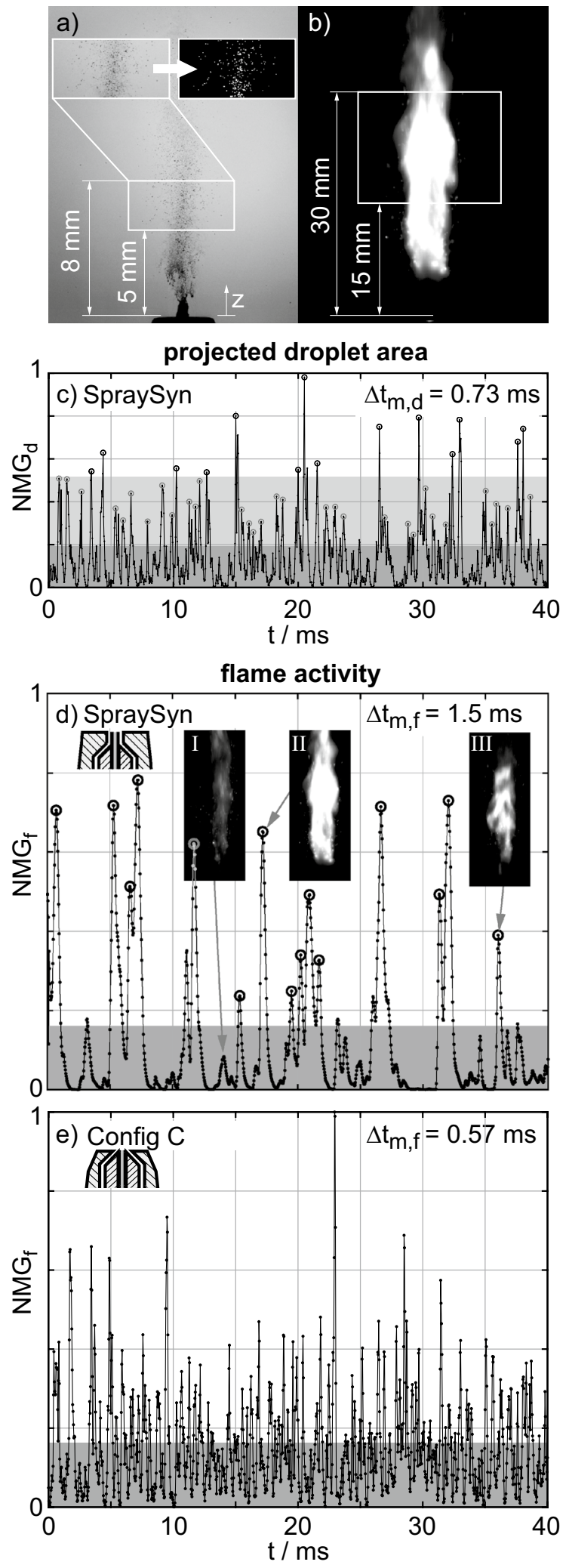

In Fig. 3c, the resulting temporal distribution of NMG for projected droplets is presented for a short exemplary time interval. Therein, high NMG values are an indicator 
for high amounts of liquid in the evaluated image section. The overall arithmetic mean signal amplitude is low at $\mathrm{NMG}_{\mathrm{m}, \mathrm{d}}=0.17$ compared to the highest peak value that is normalized to $\mathrm{NMG}_{\mathrm{d} \text {, max }}=1$. Randomly distributed high peaks indicate large lumps of liquid being shed from the initial liquid bulk at nozzle exit. Determination of specific shedding frequencies by means of an FFT analysis leads to a broad distribution of frequencies without a distinct peak due to the non-harmonic behavior and noise. Instead, a mean time interval $\Delta \mathrm{t}_{\mathrm{m}, \mathrm{d}}$ can be determined that represents the average time interval between two consecutive peaks. To determine $\Delta \mathrm{t}_{\mathrm{m}, \mathrm{d}}$, a "find-peak" algorithm is applied. To attenuate noise, two thresholds are utilized as input values for the algorithm: i) minimum time interval distance of two consecutive peaks $\Delta \mathrm{t}_{\text {min,d }}=10 /$ fps and ii) minimum peak amplitude threshold. The latter is set to the arithmetic mean value of the data signal $\left(\mathrm{NMG}_{\mathrm{min}, \mathrm{d}}=\mathrm{NMG}_{\mathrm{m}, \mathrm{d}}\right)$, indicated by the dark-grey shaded background in Fig. 3c and d. To determine the threshold for minimum time interval distance, a sensitivity analysis is conducted, by means of evaluating the gradient of the resulting characteristic mean time interval $\Delta \mathrm{t}_{\mathrm{m}, \mathrm{d}}\left(\Delta \mathrm{t}_{\text {min,d }}\right)$ over $\Delta \mathrm{t}_{\text {min,d }}$. The final threshold $\Delta \mathrm{t}_{\mathrm{m}, \mathrm{d}}$ is chosen where the gradient reached a minimum. Identified peaks are highlighted with circles as shown in Fig. 3c and d. Based on the find peak analysis, it can be concluded that the liquid shedding and consequently the detected droplet surface area fluctuates with a characteristic mean time interval of $\Delta \mathrm{t}_{\mathrm{m}, \mathrm{d}}=0.73 \mathrm{~ms}$.

Analogous to integral fluctuations of free droplet surface, the flame activity is analyzed using the same "find-peak" algorithm. However, data acquisition is performed further downstream in the region between $5 \mathrm{~mm}<\mathrm{HAB}<45 \mathrm{~mm}$ $\left(7<\Delta z / D_{1}<64\right)$, i.e., height above nozzle exit at which changes in flame intensity are most significant (see Fig. 3b. The corresponding thresholds for the "find-peak" algorithm are set to $\Delta \mathrm{t}_{\text {min,f }}=10 / \mathrm{fps}$ and $\mathrm{NMG}_{\min , \mathrm{f}}=0.5 \cdot \mathrm{NMG}_{\mathrm{m}, \mathrm{f}}$. Note that in this case, the NMG can be directly evaluated from the raw image data without the necessity of previous binarization, since high values of NMG already portray high flame activity (Bieber et al. 2019).

From qualitative comparison of the NMG data signals of each case, timescales of flame activity fluctuations appear to be significantly larger than the previously presented fluctuations of projected droplet surface, which is also reflected in determined mean event time intervals. Consequently, the mean time interval between states of high flame activity is determined to $\Delta \mathrm{t}_{\mathrm{m}, \mathrm{f}}=1.5 \mathrm{~ms}$ with a maximum value of $\Delta \mathrm{t}_{\text {max,f }}=5.9 \mathrm{~ms}$. Thus, the previously made assumption of different quasi-stationary states during the time interval of a single droplet passing the flame region is supported since the residence time of a single droplet is estimated to $\Delta \mathrm{t}=0.7 \mathrm{~ms}$. As the characteristic mean time interval between states of high flame activity is twice as large, flame activity cannot be directly correlated to fluctuating liquid mass flow at nozzle exit.

Initially, this appears to be contradictory, since qualitative comparison of the video data suggests a connection between flame fluctuation and the formation of large liquid lumps from the initial jet at nozzle exit. Corresponding high-speed images show that these lumps and subsequent secondary atomization always leads to ignition of the spray phase, as soon as droplets surpass a radial position that exceeds the outer diameter of the dispersion gas. This phenomenon can be explained by considering not only frequencies but also local variations in spray pattern associated with shedding: Resulting liquid lumps or single droplets, do not always spread in the same spatial direction. Instead, they are shed with varying radial velocity share. If the radial momentum is large enough to allow the liquid to "break" through the coaxial dispersion gas stream, it ignites in the pilot flame and presumably acts as conductor between pilot flame and evaporating droplet phase. Ultimately, this leads to ignition of the entire droplet phase in case of a flammable mixture.

In Fig 3c, the occurrence of exclusively big lumps of liquid is characterized by extreme peak values of NMG. These events can be emphasized and evaluated by shifting the amplitude threshold to higher NMG values. An increased threshold to $\mathrm{NMG}_{\min , \mathrm{d}}=2.5 \cdot \mathrm{NMG}_{\mathrm{m}, \mathrm{d}}$ is indicated by the light grey background and represents shedding of large liquid lumps. As a consequence, exclusively the black peak circles are taken into account for evaluation which leads to $\Delta \mathrm{t}_{\mathrm{m}, \mathrm{d}}=1.5 \mathrm{~ms}$, i.e., the mean time interval of two consecutive flame ignitions which could be an explanation for the qualitatively observed interdependence of droplet mass and flame.

Summarizing, the coaxial $\mathrm{O}_{2}$ dispersion gas flow presumably acts as a protective barrier that pushes back the pilot flame, encapsulates the spray phase and thus prevents spray ignition. Hence, ignition depends on two different effects associated with the liquid shedding mechanism: high peaks of temporally fluctuating shed liquid volume flow and high radial momentum. For the present nozzle configuration, however, the second effect appears to predominate.

\subsection{Alternative nozzle designs}

On the basis of this knowledge, two alternative nozzle designs are tested, as shown in Fig. 4, that aim for a reduction of large-scale flame fluctuations and prevention of flame extinction.

Approach (B) aims for a reduced distance between pilot flame and spray phase. The elevated position of the nozzle exit relative to the surrounding sinter matrix enables a guaranteed injection into the fully developed pilot flame. However, apart from an improved visual access, data evaluation of measurements with identical operation 


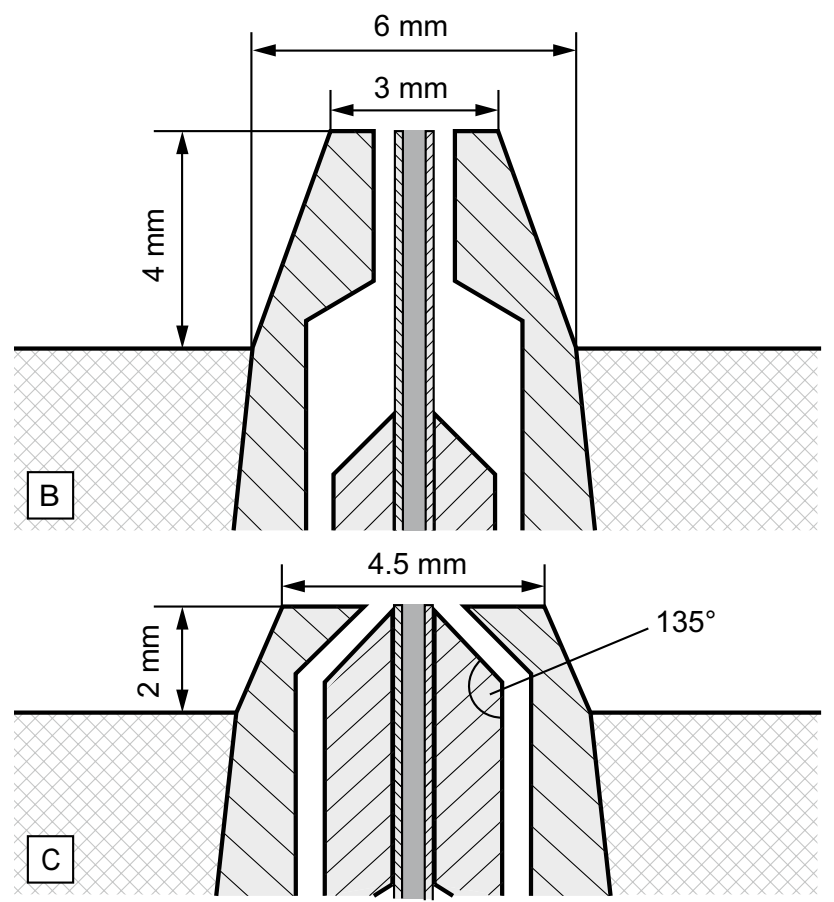

Fig. 4 B: Elevated nozzle exit position to ensure injection into fully developed pilot flame and reduced distance between pilot flame and spray; C: redirected gas flow to add radial shear to the atomization

conditions has shown no impact on timescales of flame and droplet phase fluctuations compared to the data of the original planar SpraySyn configuration (see Fig. 1); therefore, this configuration will not be further considered in the following.

The second approach C, shown in Fig. 4 bottom, also features improved visual access due to an elevated position of the nozzle exit but in addition, the gas-phase is redirected by $45^{\circ}$ at nozzle exit in order to i) reduce the axial velocity share at nozzle exit, ii) increase the average value of radial momentum. This way, the gas impingement region is shifted closer towards the nozzle exit and shearing and turbulence are increased. As a result, improved atomization and mixture formation are expected and the development of a protective $\mathrm{O}_{2}$ hollow cone barrier might be avoided. While similar configurations have already been studied in the literature (cf. e.g. Mädler et al. 2002), in Configuration C, key parameters such as characteristic length of gas and liquid flow and corresponding cross section areas remain identical to the original SpraySyn configuration. This way, comparability is ensured since identical gas and liquid mass flow rates lead to the same dimensionless quantities (e.g., Re, We) and stoichiometry at the same time, which is not the case with similar nozzle configurations in the literature. If key nozzle dimensions are changed (e.g., gap width of dispersion gas (Mädler et al. 2002), identical flow parameters $\left(\operatorname{Re}_{1}, \operatorname{Re}_{\mathrm{g}}\right)$ will lead to different Mach numbers and/or stoichiometry.

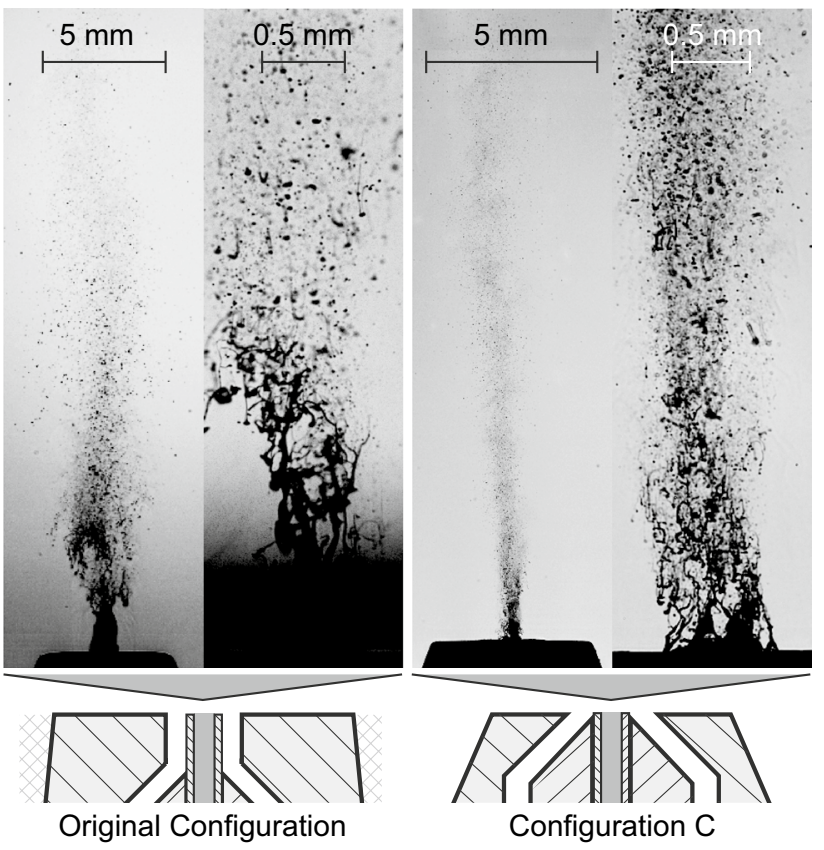

Fig.5 Qualitative comparison of primary atomization shadowgraphy images at nozzle exit for original SpraySyn (left) and modified nozzle configuration $\mathrm{C}$ (right) for identical injection parameters. The spray appears to be refined for nozzle configuration and no Schlieren structures are visible which is an indication of improved mixing at nozzle exit

In Fig. 5, a qualitative comparison of macroscopic and microscopic primary atomization shadowgraphy images at nozzle exit are presented for the original SpraySyn (left) and the modified nozzle configuration $\mathrm{C}$ (right) with identical operation conditions (see Table 1).

Compared to the SpraySyn nozzle, the resulting spray of nozzle configuration $\mathrm{C}$ appears to be characterized by more homogeneous and smaller droplet sizes. In addition, the formation of large liquid lumps is avoided. To compare the nozzle configurations quantitatively, the "find peak" algorithm (see Fig. 3) is applied for both configurations. In addition to the characteristic mean time intervals $\left(\Delta \mathrm{t}_{\mathrm{m}}\right)$, arithmetic mean values ( $\overline{\mathrm{NMG}})$ and standard deviations $(\sigma)$ normalized to the arithmetic mean value are determined and compared for both cases, i.e., droplet and flame fluctuations. In addition, the dimensionless active flame time $\left(\mathrm{t}_{\text {act. flame }}^{*}\right)$ is determined by counting all frames for which $\mathrm{NMG}_{\mathrm{f}}>=0.5 \cdot \overline{\mathrm{NMG}}_{\mathrm{f}}$ applies and subsequent normalization with the total number of frames. In Table 2, the calculated data regarding the projected droplet area and flame activity of the SpraySyn nozzle configuration and the modified configuration $\mathrm{C}$ are compared.

The nozzle modification significantly decreases the characteristic mean time interval between high flame activity $\left(\Delta \mathrm{t}_{\mathrm{m}, \mathrm{f}}\right)$, which indicates the avoidance of unwanted largescale fluctuations (cf. Fig. 3, e). In addition, the flame 
Table 2 Comparison of characteristic mean time intervals between adjacent peaks, arithmetic mean value, median and normalized standard deviation of NMG for the SpraySyn nozzle configuration and the modified configuration $\mathrm{C}$

\begin{tabular}{llllll}
\hline & \multicolumn{2}{l}{ Droplet data } & & \multicolumn{2}{l}{ Flame data } \\
Config. & SpraySyn & $\mathrm{C}$ & & SpraySyn & $\mathrm{C}$ \\
\hline$\Delta \mathrm{t}_{\mathrm{m}}(\mathrm{ms})$ & 0.73 & 0.52 & & 1.5 & 0.57 \\
$\overline{\mathrm{NMG}}(-)$ & 0.17 & 0.20 & & 0.16 & 0.20 \\
$\mathrm{NMG}(-)$ & 0.12 & 0.19 & & 0.078 & 0.174 \\
$\sigma(\%)$ & 90.78 & 45.28 & & 119.04 & 62.64 \\
$\mathrm{t}_{\text {act. flame }}^{*}(\%)$ & - & - & & 50.11 & 78.05 \\
\hline
\end{tabular}

and spray characteristic mean time intervals follow the previously expected trend and show very similar values $\left(\Delta \mathrm{t}_{\mathrm{m}, \mathrm{d}} \approx 0.52 \mathrm{~ms}\right.$ and $\left.\Delta \mathrm{t}_{\mathrm{m}, \mathrm{f}} \approx 0.57 \mathrm{~ms}\right)$. Corresponding highspeed videos of the resulting flame support the assumption that the flame is significantly stabilized by the modified nozzle configuration $\mathrm{C}$, due to visible flame activity in every frame. In addition, the share of flame active time is increased from $50.11 \%$ to $78.05 \%$. Hence, it can be assumed for configuration $\mathrm{C}$ that fluctuations in flame activity are influenced by the following two remaining effects: i) gas-phase turbulence or ii) fluctuations of the available free reactive surface. The fact that the modified nozzle leads to more stable flame operation can also be shown by analysing the medians and standard deviations. For configuration C, the median (NM̃G) of the NMG for both, flame and droplets, is very close to corresponding arithmetic mean values ( $\overline{\mathrm{NMG}})$, but deviates considerably from the arithmetic mean for the SpraySyn configuration. Accordingly, the standard deviations of the SpraySyn configuration are approximately equal to the arithmetic mean, while for configuration $\mathrm{C}$, the standard deviation is only approximately half of the arithmetic mean.

\subsection{Influence of nozzle design on spray characteristics}

In Fig. 6, quantitative spray and particle measurement data of the SpraySyn nozzle configuration (circle-shape) and the improved configuration $\mathrm{C}$ (rhombus-shape) are presented in dependency on HAB. Left, the axial velocity (red) and Sauter mean droplet diameter (blue) are shown for $0 \mathrm{~mm}<\mathrm{HAB}<50 \mathrm{~mm}$ and right, the corresponding Sauter mean mobility particle diameter (green) is presented for $50 \mathrm{~mm}<\mathrm{HAB} \leq 50 \mathrm{~mm}$. Starting at the nozzle exit, droplet sizes (blue lines) are decreasing due to a combination of evaporation and secondary atomization processes until they are increasing again from $\mathrm{HAB}>15 \mathrm{~mm}$, since small droplets are already completely evaporated while bigger droplets are still present (Bieber et al. 2019).

This characteristic shape matches well with the corresponding velocity distribution. Towards $\mathrm{HAB}=15 \mathrm{~mm}$, the mean axial velocity increases, due to droplet acceleration, until it decreases again, due to air entrainment and an increasing cross sectional flow area.

The Sauter mean droplet diameter distributions along the center axis represent a quantification of the qualitative observation of refined spray with nozzle configuration $\mathrm{C}$ (cf. Fig. 4). While both distributions have a similar characteristic shape, mean droplets are smaller for configuration $\mathrm{C}$ with a reduced diameter range as shown in corresponding exemplary droplet size histograms for $\mathrm{HAB}=40 \mathrm{~mm}$. Overall, relative velocity and thus shearing are increased at nozzle exit, which leads to an increased grade of atomization.

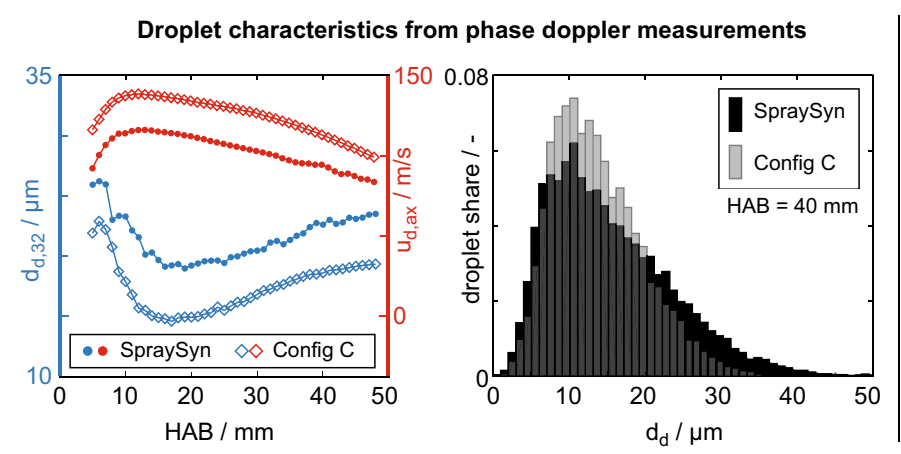

Fig. 6 Spray data: Comparison of mean axial droplet velocity profile $\mathrm{u}_{\mathrm{ax}}$ (red) and Sauter mean diameter profile $\mathrm{d}_{\mathrm{d}, 32}$ (blue) along the center axis, both obtained from PDA measurements. Complementary droplet size distributions are presented for each nozzle type exemplarily at $\mathrm{HAB}=40 \mathrm{~mm}$. Particle data: Particle electric mobility diameter $d_{\text {em, } 10}$ over HAB, determined with SMPS (green). Comple-

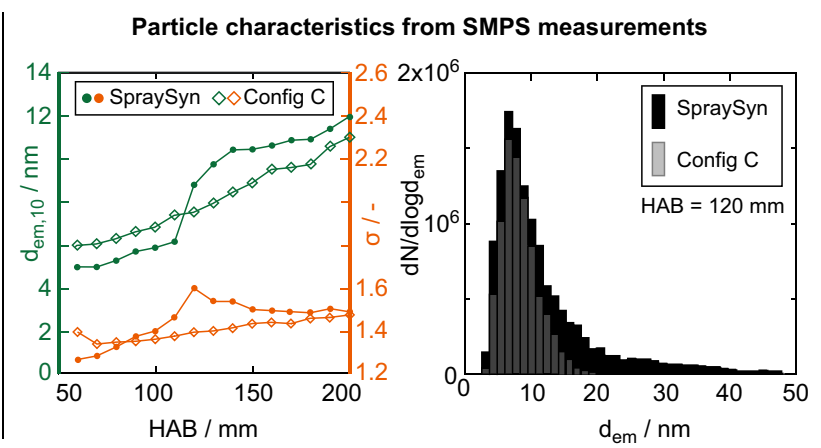

mentary particle size distributions are presented for each nozzle type exemplarily at $\mathrm{HAB}=120 \mathrm{~mm}$. All presented data are obtained with standard SpraySyn operating conditions $\left(2 \mathrm{ml} / \mathrm{s}-0.1 \mathrm{~mol} / 1 \mathrm{Fe}\left(\mathrm{NO}_{3}\right)_{3}\right.$ in $65 / 35 \%$ ethylhexanoic acid-ethanol mixture, $10 \mathrm{slm} \mathrm{O}_{2}$ dispersiongas flow) for both nozzle configurations 
Table 3 Comparison of TGA-MS and BET particle data for original SpraySyn nozzle and configuration C. During the TGA mass loss, the emission of mass sizes matching to $\mathrm{CO}_{2}$ was detected by the MS

\begin{tabular}{lll}
\hline & SpraySyn & C \\
\hline TGA & & \\
Weight loss (mass\% & 17.3 & 13.3 \\
BET & & \\
SSA $\left(\mathrm{m}^{2} / \mathrm{g}\right)$ & 195 & 290 \\
$\mathrm{~d}_{\mathrm{P}, \mathrm{BET}}(\mathrm{nm})$ & 5.9 & 3.9 \\
\hline
\end{tabular}

\subsection{Influence of nozzle design on particle structure evolution}

Since the nozzle variation leads to differences in atomization, mixture formation, and flame activity, an influence of the nozzle geometry on particle characteristics was expected. Thus, syntheses are performed with both nozzles (SpraySyn and configuration C) and final powder properties are determined by BET and TGA-MS measurements (Table 3). Additionally, samples are extracted by utilization of a HIAT probe at various HAB in situ (Fig. 6). Our investigations have shown that particle structure properties as well as the elemental composition of the final particle output can be influenced by solely varying the nozzle geometry.

The production of particles with certain tailored structure properties is one major challenge in the field of FSP synthesis. Our study indicated that the agglomerate size and the primary particle sizes are sensitive to the nozzle geometry. Physisorption measurements by BET method revealed the powders specific surface area (SSA). The average primary particle size $d_{P}$ was estimated subsequently by assuming the presence of ideal spherical, monodisperse primary particles. In gas phase synthesis, the development of primary particle sizes mainly takes place during the particle residence in high-temperature regions within the flame (Gröhn et al. 2014). Thus, $d_{p}$ can reveal information regarding differences in the initial particle growth by collision and sintering in the hot flame regions. The powder comparison revealed strong differences for both nozzles, as the SSA for nozzle configuration $\mathrm{C}\left(290 \mathrm{~m}^{2} / \mathrm{g}\right)$ significantly exceeds the value for the SpraySyn case $\left(195 \mathrm{~m}^{2} / \mathrm{g}\right)$. By assuming a bulk density of $5.24 \mathrm{~g} / \mathrm{cm}^{3}\left(\gamma-\mathrm{Fe}_{2} \mathrm{O}_{3}\right.$ as product output), the mean primary particle size can be calculated to $5.9 \mathrm{~nm}$ for the SpraySyn nozzle and $3.9 \mathrm{~nm}$ for configuration $\mathrm{C}$. Therefore, configuration $\mathrm{C}$ leads to a reduced sintering dominance which can be argued qualitatively by time averaged average spray/ flame characteristics. By comparing the flame shape and mean droplet velocities for both nozzles, one can roughly estimate differences in the particles residence times in the hot flames. Our study has shown that SpraySyn produces a larger flame while droplets move with a lower axial velocity
Table 4 Comparison of flame height (h), average droplet velocity of all droplets $\left(\overline{\mathrm{u}}_{\mathrm{d}}\right)$, all droplets smaller than $4 \mu \mathrm{m}\left(\overline{\mathrm{u}}_{\mathrm{d} \leq 4 \mu \mathrm{m}}\right)$ along the center line and corresponding calculated droplet residence time $\left(\Delta t_{\text {res } \leq 4 \mu \mathrm{m}}\right)$ for original SpraySyn nozzle and configuration $C$ as an indicator for maximum particle residence time in each flame

\begin{tabular}{lll}
\hline Configuration & SpraySyn & $\mathrm{C}$ \\
\hline $\mathrm{h}(\mathrm{mm})$ & 73 & 63 \\
$\overline{\mathrm{u}}_{\mathrm{d}}(\mathrm{m} / \mathrm{s})$ & 104.70 & 125.26 \\
$\overline{\mathrm{u}}_{\mathrm{d} \leq 4 \mu \mathrm{m}}(\mathrm{m} / \mathrm{s})$ & 109.16 & 131.47 \\
$\Delta \mathrm{t}_{\mathrm{res} \leq 4 \mu \mathrm{m}}(\mu(\mathrm{s}))$ & 669.74 & 479.20 \\
\hline
\end{tabular}

(ref. Table 4). Calculated maximum residence time of small droplets $(\leq 4 \mu \mathrm{m})$ is significantly larger for the SpraySyn nozzle compared to configuration $\mathrm{C}\left(\Delta \mathrm{t}_{\mathrm{res}, \text { Spraysyn }}=670 \mu \mathrm{s}\right.$, $\left.\Delta \mathrm{t}_{\mathrm{res}, \mathrm{C}}=479 \mu \mathrm{s}\right)$. Assuming that the particle residence time correlates qualitatively to droplet residence times, configuration $\mathrm{C}$ potentially reduces particle sintering. However, further investigation of flame temperature fields is necessary for a more detailed investigation of the nozzle design influence on the particle sintering.

Although the BET values match qualitatively to the comparison of the particle residence time in hot flame regions, we want to emphasize that the BET values should be reviewed critically for two reasons. First, BET reveals a surface equivalent, volume-related average particle size. However, due to flame pulsations, different synthesis conditions are temporally apparent and a certain (unknown) polydispersity in the final product has to be expected in general. Secondly, during FSP, particle precipitation within the hot droplet phase can be present, leading to the production of larger, dense and nearly spherical particles, which is referred to as droplet to particle-pathway. This side pathway can lead to a second particle mode, ranging from the two digit to three digit nm regime (Strobel and Pratsinis 2011; Jossen et al. 2005). Both cases lead to an unknown degree of polydispersity within the product, and in particular, the latter one may affect the physisorption measurements sensitively. At this point, we want to emphasize that there is still a lack of data in literature describing the exact (mass) ratio of droplet originated particles in respect to gas borne particles. Third, TGA-MS measurements indicate the presence of an unneglectable amount of carbon in the final powder outcome for both configurations. Product impurities may affect the physisorption measurement as well. However, the potential origin of carbon residuals as detected from TGA-MS measurement is described in the next section.

Beside the effect on the primary particle size evolution, it was shown that the change in the nozzle design also influences the evolution of resulting particle agglomerate/aggregate sizes. By means of a HIAT sampling probe, particle ensembles were diluted in situ and particle size distributions were determined by SMPS measurements (Tischendorf et al. 
2020). Along the center line between 5 to $11 \mathrm{~cm} \mathrm{HAB}$, a linear raise in the mobility diameter can be observed. In this area configuration $C$ leads to larger particle diameter which is in accordance to the BET results. Thus, configuration $\mathrm{C}$ may lead to reduced sintering and smaller primary particle sizes, but their agglomeration leads consequently to the evolution of larger and likely more fractal aggregates and agglomerates. The linear raise of the particle diameter is an indication that particle growth is already solely dominated by agglomeration/aggregation Tsantilis and Pratsinis (2004). It is likely that the primary particle size evolution by collision and sintering might be finished already at lower HAB. Interestingly, from $12 \mathrm{~cm} \mathrm{HAB}$ and above the SpraySyn nozzle leads to the formation of larger particles and the corresponding size distributions are broadened due to the presence of particle sizes up to $50 \mathrm{~nm}$ (Fig. 6). The origin of those larger particles is not yet fully understood. However by transmission electron microscopy of in situ extracted particle samples, it was revealed earlier that visibly larger and partially sintered aggregates evolve in regions above the flame tip, presumably as a result of particle re-circulation (Tischendorf et al. 2020). At higher HAB, the standard deviations for both nozzle geometries are converging asymptotically, likely caused by the aerosols self-preservation at later synthesis stages Vemury and Pratsinis (1994), while the SpraySyn case still indicates larger agglomerates.

\subsection{Influence of nozzle design on final powder elemental composition}

During TGA-MS measurement, powders of both nozzles have been heated in air until reaching their mass steady state. The MS revealed an initial release of adsorption water, followed by an emission of $\mathrm{CO}_{2}$ until $300^{\circ} \mathrm{C}$, whereby the mass loss was higher for SpraySyn $(17,3 \%)$ in comparison with the powder obtained from synthesis with nozzle configuration $\mathrm{C}(13,3 \%)$. The emission of $\mathrm{CO}_{2}$ during the powders thermal treatment may have several origins. Organic material might be present, leading to an emission of $\mathrm{CO}_{2}$ during its oxidation under oxygen-rich air atmosphere. At one side, carbon containing material might be present as soot. Soot production might be caused by flame pulsations, since those may lead to flame states with variable flame stoichiometry, despite lean combustion operating conditions in our test setup (Tischendorf et al. 2020). On the other side, organic species of the precursor formulation might pass through the synthesis chain without fully reacting. Especially larger particles from the droplet to particle pathways might carry significant amounts of organic material, in case they do not pass high temperature regions for sufficient residence time. In this case, droplet size distributions and droplet trajectories would predominantly affect the product composition. At this point, we want to emphasize that the $\mathrm{CO}_{2}$ emission can also be present, if free Carbondioxide is integrated as bidentate at the particles surfaces (Grimm et al. 1997). However, surface bidentates are unlikely the dominant origin for the $\mathrm{CO}_{2}$ emission as the relative mass loss during the TGA does not scale with the free surface area of the powders. Although the origin of organic residuals is not clear so far, the atomization and flame characteristics do affect their quantitative presence. Therefore, flame activity and corresponding droplet characteristics seem to play important roles in the particle synthesis chain during FSP. However, to the authors knowledge they have not yet been focused and described in literature and might explain possible literature discrepancies of particle characteristics produced under similar operating conditions but with different nozzle types. For instance, ambivalent results can be found by solely comparing the values in this work to product purities derived in literature under comparable synthesis and atomization conditions. In particular, Grimm et al. 1997 produced $\gamma-\mathrm{Fe}_{2} \mathrm{O}_{3}$ under lean combustion conditions as well. However, the authors concluded organic impurities corresponding to values in the low single-digit regime mass percentage (Grimm et al. 1997). During the synthesis of Zirconium Oxides and Silanol also even highest product purities (with practically zero carbon content) have been derived in literature (Spyrogianni et al. 2017; Mueller et al. 2004). However, this point one has to mention that the evolution of product impurities might be also highly sensitive to the applied precursor composition.

\section{Conclusion}

In the context of spray-flame synthesis, flame fluctuations are associated with changes in local synthesis conditions and may lead to an uncontrollable particle properties. Such flame fluctuations are influenced in particular by the atomization processes at nozzle exit and thus by atomizer design. It is found that a nozzle with cylindrical coaxial gas flow promotes large-scale flame fluctuations.

However, evaluated high-speed images indicate that characteristic time intervals between peaks of flame activity are significantly larger than time intervals between peaks of droplet concentration. Hence, there is no direct link between temporally varying droplet concentration and flame activity. This observation is explained by a random interaction of large droplets with the coaxial dispersion gas flow, which encapsulates the spray and acts as a protective barrier between spray and pilot flame. A nozzle design with angled dispersion gas that increases radial momentum transfer (and thereby shear and turbulence) leads to improved grades of atomization and is found to be a suitable approach towards increased flame stability. Remaining fluctuations of the flame are now imposed by gas-phase turbulence or mass 
flow fluctuations and characterized by significantly reduced time intervals.

With regard to particle outcome, BET measurements show that the stabilized boundary conditions lead to increased specific surface area from 195 to $290 \mathrm{~m}^{2} / \mathrm{g}$. In addition, the arithmetic mean primary particle diameter is reduced from 5.9 to $3.9 \mathrm{~nm}$ and particle size distributions obtained from SMPS measurements show a higher share of small particles with narrow distribution. Hence, the proposed nozzle design with increased radial interaction of smaller droplets and pilot flame is a promising approach for spray-flame synthesis of nanoparticles. It enables improved boundary conditions and ultimately refined particle size output.

Acknowledgements Funded by the Deutsche Forschungsgemeinschaft (DFG, German Research Foundation) - Project Number 375857587.

Funding Open Access funding enabled and organized by Projekt DEAL.

Open Access This article is licensed under a Creative Commons Attribution 4.0 International License, which permits use, sharing, adaptation, distribution and reproduction in any medium or format, as long as you give appropriate credit to the original author(s) and the source, provide a link to the Creative Commons licence, and indicate if changes were made. The images or other third party material in this article are included in the article's Creative Commons licence, unless indicated otherwise in a credit line to the material. If material is not included in the article's Creative Commons licence and your intended use is not permitted by statutory regulation or exceeds the permitted use, you will need to obtain permission directly from the copyright holder. To view a copy of this licence, visit http://creativecommons.org/licenses/by/4.0/.

\section{References}

Andronescu E (2017) Nanostructures for drug delivery. Micro and Nano Technologies. Elsevier Science

Bieber M, Menzel S, Thiebes AL, Cornelissen CG, Jockenhoevel S, Kneer R, Reddemann MA (2017) Viability of coaxial atomization for disintegration of cell solutions in cell spray applications. In: Proceedings ILASS-Europe 2017. 28th conference on liquid atomization and spray systems, Universitat Politecnica Valencia, Valencia

Bieber M, Tischendorf R, Schmid H-J, Reddemann M, Kneer R (2019) Influence of precursor concentration on spray and particle formation in flame spray pyrolysis. In: Proceedings ILASS-Europe 2019. 29th Conference on Liquid Atomization and Spray Systems, Sorbonne University, Paris

Brunauer S, Emmett PH, Teller E (1938) Adsorption of Gases in Multimolecular Layers. J Am Chem Soc 60(2):309-319

Greenberg JB, Cohen R (1997) Dynamics of a pulsating spray-diffusion flame. J Eng Math 31:397-409

Greenberg J, McIntosh A, Brindley J (2001) Linear stability analysis of laminar premixed spray flames. Proc R Soc Lond Ser Math Phys Eng Sci 457:1-31

Grimm S, Schultz S, Barth S (1997) Flame pyrolysis - a preparation route for ultrafine pure $\mathrm{y}-\mathrm{Fe} 2 \mathrm{O} 3$ powders. J Mater Sci 32:1083-1092
Grimm S, Stelzner T, Leuthäußler J, Barth S, Heide K (1997) Particle Size Effects on The Thermal Behaviour of Maghemite Synthesised by Flame Pyrolysis. Thermochimica Acta 141-148

Gröhn AJ, Pratsinis SE, Sanchez-Ferrer A, Mezzenga R, Wegner K (2014) Scale-up of nanoparticle synthesis by flame spray pyrolysis: the high-temperature particle residence time. Ind Eng Chem Res 53:10734-10742

Harvazinski ME, Huang C, Sankaran V, Feldman T, Anderson W, Merkle C, Talley DG (2013) Combustion instability mechanisms in a pressure-coupled gas-gas coaxial rocket injector. In: 49th AIAA/ ASME/SAE/ASEE joint propulsion conference, American Institute of Aeronautics and Astronautics, Reston, Virginia

Jossen R, Pratsinis SE, Stark WJ, Mädler L (2005) Criteria for flamespray synthesis of hollow, shell-like, or inhomogeneous oxides. J Am Ceram Soc 88:1388-1393

Kammler HK, Mädler L, Pratsinis SE (2001) Flame Synthesis of Nanoparticles. Chem Eng Technol 24:583-596

Lasheras JC, Hopfinger EJ (2000) Liquid jet instability and atomization in a coaxial gas stream. Annu Rev Fluid Mech 32:275-308

Lasheras JC, Villermaux E, Hopfinger EJ (1998) Break-up and atomization of a round water jet by a high-speed annular air jet. J Fluid Mech 357:351-379

Li S, Ren Y, Biswas P, Tse SD (2016) Flame aerosol synthesis of nanostructured materials and functional devices: Processing, modeling, and diagnostics. Process Energy Combust Sci 55:1-59

Marmottant P, Villermaux E (2004) On spray formation. J Fluid Mech 498:73-111

Meierhofer F, Hodapp M, Achelis L, Buss L, Noriler D, Meier HF, Fritsching U (2014) Untersuchung von Zerstäubungskonzepten für erhöhte Produktionsraten der Flammensprühpyrolyse (FSP). Materialwiss Werkstofftech 8:765-777

Menser J, Kluge S, Wiggers H, Dreier T, Schulz C (2015) Approach to standardize s spray-flame nanoparticle synthesis burner. In: Proceedings of the European Combustion Meeting, European Combustion Meeting 7, Budapest

Mueller R, Jossen R, Kammler HK, Pratsinis SE, Akhtar MK (2004) Growth of zirconia particles made by flame spray pyrolysis. AIChE J 50(12):3085-3094

Mädler L, Kammler HK, Mueller R, Pratsinis SE (2002) Controlled synthesis of nanostructured particles by flame spray pyrolysis. J Aerosol Sci 33:369-389

Pitcher G, Wiley G (1991) Sensitivity of dropsize measurements by phase doppler anemometry to refractive index changes in combusting fuel sprays. Applications of Laser Techniques 227-247

Schneider F, Suleiman S, Menser J, Borukhovich E, Wlokas I, Kempf A, Wiggers H, Schulz C (2019) SpraySyn: a standardized burner configuration for nanoparticle synthesis in spray flames. Rev Sci Instrum 90:85-108

Sokolowski M, Sokolowska A, Michalski A, Gokieli B (1977) The "InFlame-Reaction" Method for $\mathrm{Al}_{2} \mathrm{O}_{3}$ Aerosol Formation. J Aerosol Sci 8:219-230

Spyrogianni A, Herrmann IK, Keevend K, Pratsinis SE, Wegner K (2017) The silanol content and in vitro cytolytic activity of flamemade silica. J Colloid Interface Sci 507:95-106

Stark WJ, Pratsinis SE (2002) Aerosol flame reactors for manufacture of nanoparticles. Powder Technol 126:103-108

Strobel R, Pratsinis SE (2007) Flame aerosol synthesis of smart nanostructured materials. J Mater Chem 17:4743-4756

Strobel R, Pratsinis SE (2011) Effect of solvent composition on oxide morphology during flame spray pyrolysis of metal nitrates. Phys Chem Chem Phys 11:9246-9252

Teoh WY, Amal R, Mädler L (2010) Flame spray pyrolysis: an enabling technology for nanoparticles design and fabrication. Nanoscale 2:1324-1347

Thiebaut B (2011) Flame spray pyrolysis: a unique facility for the production of nanopowders. Platin Met Rev 55:149-151 
Tischendorf R, Simmler M, Weinberger C, Bieber M, Reddemann MA, Fröde F, Lindner J, Pitsch H, Kneer R, Tiemann M, Nirschl H, Schmid H-J (2020) Examination of the evolution of iron oxide nanoparticles in flame spray pyrolysis by tailored in situ particle sampling techniques. Journal of aerosol science 154

Tsantilis S, Pratsinis SE (2004) Soft- and hard-agglomerate aerosols made at high temperatures. ACS J Surf Colloids 20(14):5933-5939

Varga CM, Lasheras JC, Hopfinger EJ (2003) Initial breakup of a small-diameter liquid jet by a high-speed gas stream. J Fluid Mech 497:405-434
Vemury S, Pratsinis SE (1994) Self-preserving size distributions of agglomerates. J Aerosol Sci 26(2):175-185

Publisher's Note Springer Nature remains neutral with regard to jurisdictional claims in published maps and institutional affiliations. 